NISTIR 5015

\title{
CROSSTALK BETWEEN MICROSTRIP TRANSMISSION LINES
}

David A. Hill Kenneth H. Cavcey Robert T. Johnk

QE 100 .456 \#5015 1993 



\section{CROSSTALK BETWEEN MICROSTRIP TRANSMISSION LINES}

David A. Hill

Kenneth H. Cavcey

Robert T. Johnk

Electromagnetic Fields Division Electronics and Electrical Engineering Laboratory National Institute of Standards and Technology Boulder, Colorado 80303-3328

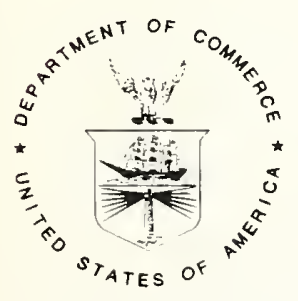


Abstract. . . . . . . . . . . . . . . . . . . . . . . .

1. INTRODUCTION . . . . . . . . . . . . . . . . . . . . .

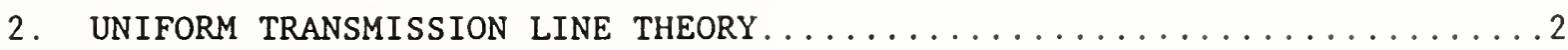

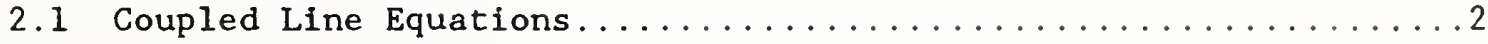

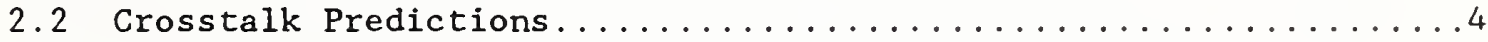

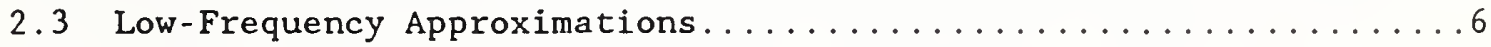

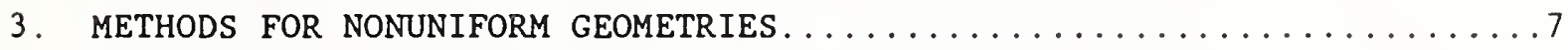

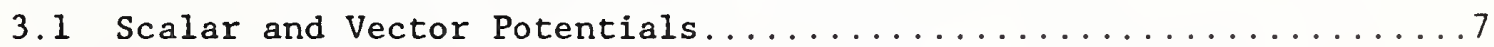

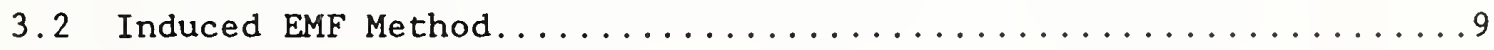

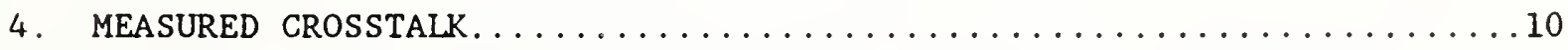

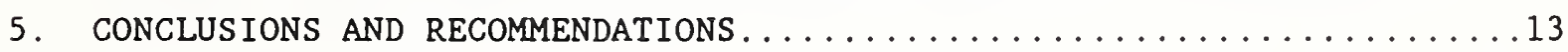

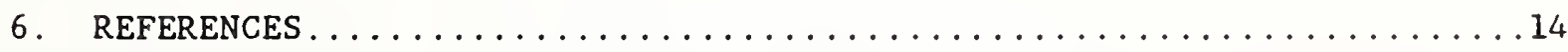

APPENDIX A. EQUIVALENCE OF POTENTIAL AND TRANSMISSION LINE THEORIES....16 APPENDIX B. TRANSMISSION LINE PARAMETERS FOR CIRCULAR WIRES ........19

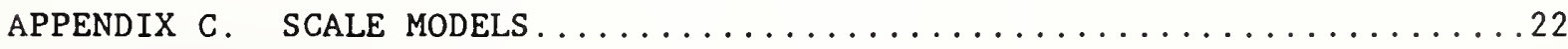



David A. Hill, Kenneth H. Cavcey, and Robert T. Johnk

Electromagnetic Fields Division

National Institute of Standards and Technology

Boulder, CO 80303

\begin{abstract}
Methods for prediction of crosstalk between microstip transmission lines are reviewed and simplified for the weak coupling case. Classical coupled transmission line theory is used for uniform lines, and potential and induced EMF methods are used for crosstalk between nonuniform lines. It is shown that the potential method is equivalent to classical coupled transmission line theory for the case of uniform lines. An experiment was performed for uniform coupled microstrip lines for frequencies from $50 \mathrm{MHz}$ to $5 \mathrm{GHz}$, and good agreement between theory and measurement was obtained for both near-end and farend crosstalk.
\end{abstract}

Key words: characteristic impedance; crosstalk; effective permittivity; even mode; microstrip; mutual capacitance; mutual inductance; odd mode; scalar potential; transmission line; vector potential.

\title{
1. INTRODUCTION
}

In dense circuits, electromagnetic coupling (crosstalk) between closely spaced signal lines limits interconnect performance [1] and becomes an important aspect of circuit design [2]. The general crosstalk problem involves multiple lines and complex geometries and is very complicated to analyze. In this report we analyze crosstalk between a pair of microstrip lines located on a single grounded substrate. This geometry is simple enough to permit analysis, but still illustrates most of the important features of the crosstalk issues.

The organization of this report is as follows. Section 2 starts with a review of uniform transmission line theory for a pair of coupled lines and goes on to obtain simple expressions for near-end and far-end crosstalk [3]. Section 3 covers two perturbation methods [4,5] that are valid for arbitrary line orientations, but require that the coupling be weak (multiple interactions are neglected). Section 4 presents a comparison of measured 
and calculated results for the $S$ parameters over a broad frequency range for a pair of uniform microstrip lines. Section 5 contains conclusions and recommendations for further study. Appendix A demonstrates the equivalence of the potential method in Section 3.1 and transmission line theory in

Section 2 for the case of uniform, weakly coupled lines. Appendix B contains a derivation of the transmission line parameters for the simple example of circular wires above a ground plane. Appendix $C$ discusses length and frequency scaling of lossless and lossy transmission lines.

\section{UNIFORM TRANSMISSION LINE THEORY}

\subsection{Coupled Line Equations}

The literature on crosstalk between transmission lines dates back at least to the 1930s [6], and textbooks have been written on multiconductor transmission lines [7,8]. Strictly speaking, classical transmission line theory applies only to perfectly conducting lines in a homogeneous medium so that the transmission line modes are transverse electromagnetic (TEM). Microstrip lines do not support pure TEM modes, but at low frequencies they support quasi-TEM modes [9] that approximately satisfy the transmission line equations.

A cross-sectional view of a pair of microstrip lines on a grounded substrate is shown in figure 1. For simplicity, we assume that the two strips have equal width w, zero thickness, and perfectly conductivity. (We will comment on the effects of conductor losses later.) The ground plane is also assumed to be perfectly conducting. The lines are located on a dielectric slab (substrate) of thickness h and have a separation s. The substrate has relative permittivity $\epsilon_{\mathrm{r}}$ and free-space permeability $\mu_{0}$. The region above the substrate is free space.

The multiconductor transmission line equations can be compactly written in matrix form [10], but for discussion we choose to write out the coupled differential equations. For the source-free case, the line currents, $I_{1}$ and $I_{2}$, and voltages, $V_{1}$ and $V_{2}$, satisfy [3] 


$$
\begin{aligned}
& \frac{d V_{1}}{d x}+j \omega L_{11} I_{1}=-j \omega L_{12} I_{2}, \\
& \frac{d I_{1}}{d x}+j \omega C_{11} V_{1}=-j \omega C_{12} V_{2}, \\
& \frac{d V_{2}}{d x}+j \omega L_{22} I_{2}=-j \omega L_{21} I_{1}, \\
& \frac{d I_{2}}{d x}+j \omega C_{22} V_{2}=-j \omega C_{21} V_{1},
\end{aligned}
$$

where $x$ is the longitudinal coordinate and the $\exp (j \omega t)$ time dependence is suppressed. The $\mathrm{C}_{i j}$ are the elements of the distributed capacitance matrix, and the $L_{i j}$ are the elements of the distributed inductance matrix [11]. Both the capacitance and inductance matrices are symmetric $\left(C_{12}=C_{21}\right.$ and $\left.\mathrm{L}_{12}=\mathrm{L}_{21}\right)$. Because of the microstrip symmetry, we also have $\mathrm{C}_{11}=\mathrm{C}_{22}$ and $\mathrm{L}_{11}=\mathrm{L}_{22}$. We have put the off-diagonal terms on the right sides of the equations because they will be thought of as source terms for loosely coupled lines in Section 3.

For perfect conductors in a homogeneous dielectric, the capacitance and inductance matrices are frequency independent. When the dielectric region is inhomogeneous (as for insulated wires [11] or microstrips [12]), then the capacitance and inductance matrices depend on frequency. However, they are approximately frequency independent over a large quasi-static frequency range $[13]$.

The symmetric microstrip supports an even mode with $V_{1}=V_{2}$ and an odd mode with $V_{1}=-V_{2}$. The even and odd mode propagation constants, $\gamma_{\text {ev }}$ and $\gamma_{\text {odd }}$, are given by [3]

$$
\gamma_{e v}=j \omega\left[\left(L_{11}+L_{12}\right)\left(C_{11}+C_{12}\right)\right]^{1 / 2}
$$

and 


$$
\gamma_{\text {odd }}=j \omega\left[\left(L_{11}-L_{12}\right)\left(C_{11}-C_{12}\right)\right]^{1 / 2} .
$$

The even and odd mode characteristic impedances, $z_{e v}$ and $z_{o d d}$ are given by [3]

$$
z_{e v}=\left[\left(L_{11}+L_{12}\right) /\left(C_{11}+C_{12}\right)\right]^{1 / 2}
$$

and

$$
z_{\text {odd }}=\left[\left(L_{11}-L_{12}\right) /\left(C_{11}-C_{12}\right)\right]^{1 / 2} .
$$

Equations (2) and (3) are deceptively simple because computation of the $L_{i j}$ and $C_{i j}$ elements generally requires some numerical method, such as the method of moments [12].

For large spacing ( $\mathrm{s} / \mathrm{w} \gg 1)$, the coupling capacitance $\mathrm{C}_{12}$ and inductance $\mathrm{L}_{12}$ become small. In this case, the propagation constants in eq (2) approach that of an isolated line $\gamma_{0}$ :

$$
\gamma_{0}=j \omega\left(L_{11} C_{11}\right)^{1 / 2}
$$

Also, the characteristic impedances in eq (3) approach that of an isolated line $\mathrm{Z}_{0}$ :

$$
\mathrm{z}_{0}=\left(\mathrm{L}_{11} / \mathrm{C}_{11}\right)^{1 / 2}
$$

\subsection{Crosstalk Predictions}

To study crosstalk, we consider the geometry in figure 2. The coupled microstrip lines are identical to those in figure 1 except that they are of finite length $\ell$. Line 1 is fed with a voltage generator $v_{0}$ at $x-0$, and 
all four ports are terminated with an impedance $z_{0}$. We label the driven and terminated ends of line 1 as ports 1 and 2, and the near and far ends of line 2 as ports 3 and 4 . The geometry in figure 2 has been analyzed for both directional coupler applications [13] and crosstalk predictions [3].

For crosstalk prediction, we can assume that the lines are loosely coupled ( $s$ is not too small compared to $h$ and $w$ ). In this case, we can use the approximate solution of [3] and equate near-end and far-end crosstalk to the $\mathrm{S}$ parameters as follows:

$$
s_{31}=v_{2}(0) / v_{1}(0) \text { and } s_{41}=v_{2}(l) / v_{1}(0)
$$

In terms of the microstrip parameters, $\mathrm{s}_{31}$ is approximately [3]

$$
\mathrm{S}_{31}=\frac{\delta \mathrm{Z}}{2 \mathrm{Z}_{0}}\left\{1-\mathrm{e}^{-2 \gamma_{0} \ell}\left[\cos (2 \delta \mathrm{k} \ell)+\frac{\delta \mathrm{Z}}{\mathrm{Z}_{0}} \sin (2 \delta \mathrm{k} \ell)\right]\right),
$$

where

$$
\delta Z=\left(Z_{\text {ev }}-Z_{\text {odd }}\right) / 2 \approx \frac{j \omega}{2 \gamma_{0}}\left(L_{12}-C_{12} Z_{0}^{2}\right)
$$

and

$$
\delta \mathrm{k}=\left(\gamma_{\mathrm{ev}}-\gamma_{\mathrm{odd}}\right) /(2 \mathrm{j}) \approx \omega\left(\mathrm{L}_{12}+\mathrm{C}_{12} \mathrm{z}_{0}^{2}\right) /\left(2 \mathrm{z}_{0}\right)
$$

Similarly, $\mathrm{S}_{41}$ is approximately

$$
s_{41}=-j e^{-\gamma_{0} \ell} \sin (\delta k \ell) .
$$

(Reference [3] has the correct exact expression for $\mathrm{s}_{41}$, but has an error in the weak coupling approximation for $S_{41}$ and does not agree with eq (10).) The transmission $S$ parameter $S_{21}$ is not needed for crosstalk prediction, but is approximately [3] 


$$
\mathrm{S}_{21}=\mathrm{e}^{-\gamma_{0} \ell} \cos (\delta \mathrm{k} \ell) .
$$

To first order in $\delta Z$, the reflection coefficient $S_{11}=0$. To first order in $\delta Z$, the approximate $S$ parameters satisfy conservation of power:

$$
\left|s_{11}\right|^{2}+\left|s_{21}\right|^{2}+\left|s_{31}\right|^{2}+\left|s_{41}\right|^{2} \approx 1
$$

\subsection{Low-Frequency Approximations}

At sufficiently low frequencies (or for sufficiently short lines), we can assume that $\left|\gamma_{0} \ell\right| \ll 1$. In that case the scattering parameters of the previous section reduce to

$$
\begin{aligned}
& \mathrm{s}_{21} \approx 1, \\
& \mathrm{~s}_{31} \approx \frac{\delta \mathrm{Z}_{0} \ell}{\mathrm{Z}_{0}},
\end{aligned}
$$

and

$$
\mathrm{s}_{41} \approx-\mathrm{j} \delta \mathrm{k \ell} \text {. }
$$

To simplify the crosstalk parameters even further, we write the three propagation constants in terms of effective dielectric constants:

$$
\begin{aligned}
& \gamma_{0}=j \omega \epsilon_{\mathrm{eff}}^{1 / 2} / \mathrm{c}, \\
& \gamma_{\mathrm{ev}}=j \omega \epsilon_{\mathrm{ev}}^{1 / 2} / \mathrm{c},
\end{aligned}
$$

and

$$
\gamma_{\text {odd }}=j \omega \epsilon_{\text {odd }}^{1 / 2} / c
$$


where $c$ is the velocity of light in free space. The relative effective dielectric constant $[9,13]$ for an isolated strip is $\epsilon$ eff, and the relative effective dielectric constants for the even and odd modes [13] are $\epsilon_{\mathrm{ev}}$ and $\epsilon$ odd. Substituting eq (14) into eq (13), we obtain

$$
S_{31} \approx j \omega \frac{\epsilon \frac{1 / 2}{e f f Z}}{c Z_{0}}
$$

and

$$
S_{41} \approx-j \omega \frac{\left(\epsilon_{e v}^{1 / 2}-\epsilon_{o d d}^{1 / 2}\right) l}{2 c} .
$$

Since $\epsilon$ eff' ' $\mathrm{ev}$ ' $\epsilon$ odd' and $\mathrm{Z}_{0}$ are frequency independent for low frequencies, $\mathrm{S}_{31}$ and $\mathrm{S}_{41}$ are proportional to $j \omega$ at low frequencies. Paul and Everett [14] have shown this for the same geometry, and they showed experimentally that the resultant crosstalk waveforms are proportional to the time derivative of the excitation waveform (when the spectrum of the excitation is sufficiently band limited). Equations (15a) and (15b) also show that the crosstalk is proportional to line length $\ell$ for short lines.

\section{METHODS FOR NONUNIFORM GEOMETRIES}

The general crosstalk application will involve many lines and nonuniform geometries. For simplicity we consider only two lines on a single substrate, but allow the two lines to have arbitrary orientation. The two methods that we consider can be extended to multiple lines and multiple substrates.

\subsection{Scalar and Vector Potentials}

In this section, we follow the method of Howard and Dunn $[4,15]$ for weakly coupled microstrip lines. Consider two lines of arbitrary length and 
orientation located on the same substrate as in figure 3. The lines do not have to be straight, but here we assume that they are.

We first consider line 1 , the driven line, in the absence of line 2 . The current $I_{1}$ and charge $\rho_{1}$ are approximated as a line current and a line charge located at the center of the strip. The local coordinate $\zeta_{1}$ specifies the location on line 1 . The charge and current on line 1 produce a voltage $V(x, y)$ and a vector potential $\bar{A}(x, y)$ at an arbitrary point $(x, y)$ at the surface of the substrate $(z=h)$ :

$$
\mathrm{V}(\mathrm{x}, \mathrm{y})=\int_{\text {line } 1} \rho_{1}\left(\zeta_{1}\right) \mathrm{g}_{\mathrm{e}}\left(\zeta_{1} ; \mathrm{x}, \mathrm{y}\right) \mathrm{d} \zeta_{1}
$$

and

$$
\bar{A}(x, y)=\int_{1 \text { ine } 1} \hat{\zeta}_{1} I_{1}\left(\zeta_{1}\right) g_{m}\left(\zeta_{1} ; x, y\right) d \zeta_{1} .
$$

The unit vector $\hat{\zeta}_{1}$ is in the direction of line 1 . If the line is not straight, then $\hat{\zeta}_{1}$ is a function of position along the line. The functions $\mathrm{g}_{\mathrm{e}}$ and $\mathrm{g}_{\mathrm{m}}$ are Green's functions for a grounded substrate geometry that are defined and discussed in [16] and [17]. The simpler case of a ground plane geometry (no substrate) is discussed in Appendix B.

We now consider line 2 (the undriven line). The scalar potential $\mathrm{V}$ and vector potential $\bar{A}$ act as sources that excite line 2 . The derivation of the source terms resulting from $V$ and $\bar{A}$ is given in [15], and the resultant transmission line equations for the voltage $V_{2}$ and the current $I_{2}$ on line 2 are

$$
\frac{d V_{2}}{d \zeta_{2}}+j \omega L_{22} I_{2}=-j \omega A\left(\zeta_{2}\right)
$$

and

$$
\frac{d I_{2}}{d \zeta_{2}}+j \omega C_{22} V_{2}=j \omega C_{22} V\left(\zeta_{2}\right)
$$


$A$ is the component of the vector potential in the direction of line 2: $\mathrm{A}=\overline{\mathrm{A}} \cdot \hat{\zeta}_{2}$. Equations (17a) and (17b) are similar to eqs (1c) and (1d) except that the source terms on the right side are different. In Appendix A, we show that the two sets of equations are equivalent for the case of parallel, uniform lines. Paul's results for illumination of transmission lines by arbitrary external fields [10] can be shown to be equivalent to eqs (17a) and (17b) if we recognize that $V$ and $A$ are equal to integrals from the ground plane to the microstrip ( 0 to $h$ ) of the appropriate components of the electric and magnetic fields [10].

Equations (17a) and (17b) can be solved for $\mathrm{I}_{2}$ and $\mathrm{V}_{2}$ using standard methods [15]. Since the effect of line 2 on line 1 has been neglected, the solution for $\mathrm{I}_{2}$ and $\mathrm{V}_{2}$ can be considered a first-order perturbation solution that is valid for weak coupling. To check the validity of the method, one could allow the first order currents and charges on line 2 to excite line 1 and determine what spacing or geometry produces a negligible change in the currents and charges on line 1.

\subsection{Induced EMF Method}

Consider again the geometry in figure 3 where 1 ine 1 is the driven line, and its current and charge produce scalar and vector potentials as given by eqs (16a) and (16b). We can derive the electric field $\bar{E}$ produced by line 1 from [18]:

$$
\overline{\mathrm{E}}=-\nabla \mathrm{V}-\mathrm{j} \omega \overline{\mathrm{A}} \text {. }
$$

Now consider line 2 in the absence of line 1 . If we excite line 2 with a voltage source at $\zeta_{2}^{\prime}$, we can use standard transmission line theory to determine the current distribution $\mathrm{I}_{2}\left(5_{2}\right)$. We now apply the induced EMF method which has been so widely used in antenna analysis [19] to the 
illumination of line 2 by the electric field given by eq (18). The open circuit voltage $\mathrm{V}_{2 \mathrm{oc}}\left(S_{2}^{\prime}\right)$ is written as the following integral over line 2:

$$
V_{2 o c}\left(\zeta_{2}^{\prime}\right)=\int_{\text {line } 2} \frac{I_{2}\left(\zeta_{2}\right)}{I_{2}\left(\zeta_{2}^{\prime}\right)} \bar{E}\left(\zeta_{2}\right) \cdot \hat{\zeta}_{2} d \zeta_{2} .
$$

(To be complete, the integral should also extend over the terminations between the ground plane and the microstrip.) Olsen [5] has used the result in eq (19) to compute crosstalk between lossy transmission lines where the loss effect [20] has been included in both line 1 and line 2 by using the appropriate current distributions, $I_{1}\left(\zeta_{1}\right)$ and $I_{2}\left(\zeta_{2}\right)$, for lossy lines. The open circuit location $5_{2}^{\prime}$ can be chosen anywhere on 1 ine 2, but is normally chosen to be at either end since the end points are of most interest. Expressions of the form in eq (19) have appeared in other analyses of illumination of transmission lines by external fields [21,22], and Paul's analysis [10] shows that the potential formulation in eqs (17a) and (17b) is equivalent to the induced EMF formulation in eq (19).

We again point out that the result in eq (19) is a first-order perturbation result that neglects any multiple interaction between the lines and that the line-current approximation is made on both lines. Yuan and Nyquist [23] have used a full-wave perturbation theory to analyze microstrip crosstalk where they have not made the line-current approximation.

\section{MEASURED CROSSTALK}

For crosstalk measurements, we prepared a circuit board with two paralle1, uniform microstrip lines as in figure 2. The dielectric substrate was duroid with relative permittivity $\epsilon_{\mathrm{r}}=2.2$ and thickness $\mathrm{h}=1.55 \mathrm{~mm}$. The strip width $w$ was chosen to be $4.8 \mathrm{~mm}$, and this value yields a characteristic impedance of $50 \Omega$ for a single, isolated line in the quasistatic frequency range [13]. The strip separation was chosen equal to the strip width $4.8 \mathrm{~mm}$ in order to push the limit of the weak-coupling theory. 
The line length $\ell$ was chosen to be $19.6 \mathrm{~cm}$, so the lines range from electrically short at the lowest measurement frequency ( $50 \mathrm{MHz}$ ) to electrically long at the highest measurement frequency ( $5 \mathrm{GHz}$ ).

Even though our primary interest was in smaller, shorter monolithic microwave integrated circuit (MMIC) lines, the larger dimensions of our circuit board made it easier to perform measurements of $\mathrm{S}$ parameters using an automatic network analyzer. (The subject of scaling the measurements to smaller lines at higher frequencies is discussed in Appendix C.) We soldered coaxial, $50-\Omega$ connectors to each of the four ports as shown in the photograph in figure 4. In measuring the near-end $\left(\mathrm{S}_{31}\right)$ or far-end $\left(\mathrm{S}_{41}\right)$ crosstalk, we terminated the other two ports (ports 2 and 4 or 2 and 3 ) in $50-\Omega$ loads.

For comparison with the measured crosstalk data, we used the weakcoupling, uniform-line theory for $\mathrm{S}_{31}$ in eq (7) and $\mathrm{s}_{41}$ in eq (10). These theories require values for the even and odd mode characteristic impedances ( $Z_{\text {ev }}$ and $Z_{\text {odd }}$ ) and the effective dielectric constant for the even and odd modes and the isolated strip ( $\epsilon_{\mathrm{ev}}, \epsilon_{\text {odd }}$, and $\epsilon_{\text {eff }}$ ). Accurate quasi-static formulas for these quantities have been published in [13] and [24], and we used these formulas to compute the following values: $Z_{\text {ev }}=51.64 \Omega, Z_{\text {odd }}=$ $48.36 \Omega, \epsilon_{\text {ev }}=1.973, \epsilon_{\text {odd }}=1.797$, and $\epsilon_{\text {eff }}=1.881$. The formulas used to obtain these values were checked for accuracy by comparison with published curves obtained by numerical methods [13]. As expected, $\epsilon$ ev is greater than $\epsilon$ odd because the even mode field is more confined to the substrate.

Comparisons of measured and theoretical crosstalk magnitudes are shown in figures 5 and 6 for frequencies from $50 \mathrm{MHz}$ to $5 \mathrm{GHz}$ in $50 \mathrm{MHz}$ steps. The near-end crosstalk $\mathrm{S}_{31}$ in figure 5 is characterized by oscillations as predicted by eq (7). The low-frequency theory given by eq (15a) is also shown and is valid for frequencies below about $100 \mathrm{MHz}$. This theory has the $j \omega$ dependence that was discussed by Paul and Everett [14]. The agreement with the more general theory is generally good (within $2 \mathrm{~dB}$ ) for frequencies below $3 \mathrm{GHz}$. At higher frequencies, the lack of agreement could be due to dispersion, surface waves, or higher-order modes that are not taken into 
account in the theory. Connector-to-connector crosstalk might also be a factor above $3 \mathrm{GHz}$ because of the close spacing of the connectors as shown in figure 4. However, these frequencies are above the normal frequency range of use for microstrip lines of these dimensions [13]. The agreement that we have achieved is more than adequate for the application of crosstalk estimation where high accuracy is not required.

The far-end crosstalk $\left(\mathrm{S}_{41}\right)$ in figure 6 does not exhibit oscillations because the argument of the sine function in eq (10) never gets large enough to cause oscillations. (It could for longer lines or higher frequencies.) We see some minor oscillations in the measured data above $2.5 \mathrm{GHz}$, but the agreement remains within $2 \mathrm{~dB}$. We did not include the low-frequency theory of eq (15b), but it is essentially identical to the more general theory of eq (10) over the entire frequency range. Both theories show the jw dependence discussed by Paul and Everett [14].

Comparisons of measured and theoretical crosstalk phase are shown in figures 7 and 8 . The phase of $S_{31}$ in figure 7 approaches $90^{\circ}$ at low frequencies because of the $j \omega$ dependence in eq (15a). At higher frequencies, the phase contains $180^{\circ}$ jumps where the amplitude in figure 5 exhibits nulls. Above $3 \mathrm{GHz}$, the theory and measurement are no longer in agreement. The agreement is qualitatively good below $3 \mathrm{GHz}$, but the measured phase has an extra retardation, probably because of the phase shift associated with the connectors. The connector phase shift could be calibrated out, but we chose not to do so. For crosstalk applications, the amplitude results (which do not appear to be affected by the connectors) are much more important than the phase. The extra connector phase shift (which appears to be proportional to $\omega$ ) would result in a pure time delay in the time domain.

The phase of $\mathrm{S}_{41}$ in figure 8 approaches $-90^{\circ}$ at low frequencies because of the factor $-j$ in eq $(15 b)$. The $\exp \left(-\gamma_{0} \ell\right)$ factor in eq (10) yields a linear phase shift, and the $360^{\circ}$ jumps occur only to keep the phase in the range from $-180^{\circ}$ to $180^{\circ}$. Again the measured phase has an extra phase shift due to the connectors. 
We also measured the through parameter $S_{21}$, and it agreed well with eq (11). We measured $\mathrm{S}_{11}$ to check the matching of the connectors to microstrip lines, and it remained below $-30 \mathrm{~dB}$ for frequencies below $1 \mathrm{GHz}$ and below $-20 \mathrm{~dB}$ above $1 \mathrm{GHz}$. To estimate the connector-to-connector crosstalk, we soldered two connectors to the ground plane in the absence of microstrips and measured the coupling. It increased from $-87 \mathrm{~dB}$ at $50 \mathrm{MHz}$ to $-37 \mathrm{~dB}$ at $5 \mathrm{GHz}$. Consequently it could have been partly responsible for the deviation at the high frequencies in figure 5.

\section{CONCLUSIONS AND RECOMMENDATIONS}

We have reviewed theories for crosstalk between a pair of uniform or nonuniform microstrip transmission lines. We have concentrated on theories for weak coupling because that is the usual case of interest in crosstalk applications. For uniform lines, we have simplified the theory to easily computed formulas for near-end and far-end crosstalk and have also showed that both types of crosstalk have a simple jw dependence at low frequencies. We have also performed crosstalk measurements and have shown good agreement between theory and measurements for frequencies from $50 \mathrm{MHz}$ to $5 \mathrm{GHz}$. These measurements were made on a fairly large circuit board (length $\approx 20 \mathrm{~cm}$ ) which can be considered a scale model for smaller MMIC lines. Scale model issues are discussed in Appendix $C$.

Theories for nonuniform, coupled lines have also been reviewed. These theories are quite flexible and do not rely on the usual mutual capacitance and inductance parameters that are used in uniform, multiconductor transmission line theory. However, we have shown that the scalar and vector potential theory discussed in Section 3.1 is equivalent to coupled transmission line theory for the special case of uniform lines. This was done in Appendix A by demonstrating equivalence of scalar potential coupling to mutual capacitance and the equivalence of vector potential coupling to mutual inductance. This equivalence is important because it allowed us to show in Appendix $B$ that local mutual capacitance and inductance coupling 
occur over a finite length of line that is approximately equal to twice the line separation.

A number of extensions to this work would be worthwhile. Loss should be included in the theories because this is likely to be important in small MMIC lines and does not scale in a convenient way for scale model measurements, as discussed in Appendix $C$. More theoretical and experimental work should be done for complex geometries that include nonuniform lines and multiple dielectric layers. The potential and induced EMF methods discussed in Section 3 are useful for such cases, but their limitations need to be determined. Time-domain theory and measurements [25] should be pursued because of the importance of pulse crosstalk [14] in digital systems. Pulse issues could be studied directly in the time domain or by way of Fourier transforms of frequency-domain calculations and measurements.

\section{REFERENCES}

[1] Gravelle, L.B; Wilson; P.F. EMI/EMC in printed circuit boards - a literature review. IEEE Trans. Electromag. Compat., 34: 109-116; 1992.

[2] Goldfarb, M.; Platzker, A. The effects of electromagnetic coupling on MMIC design. Int.J. Microwave Millimeter Wave CAE, 1:38-47; 1991.

[3] Isaacs, J.C.; Strakhov, N.A. Crosstalk in uniformly coupled lossy transmission lines. Bel1 Sys. Tech. J., 52: 101-115; 1973.

[4] Howard, L.C; Dunn, J.M. An expression for the S-parameters of arbitrarily oriented microstrip lines. Proc. Int. IEEE MTT Symp.: 1543$1546 ; 1993$.

[5] 0lsen, R.G. A simple model for weakly coupled lossy transmission lines of finite length. IEEE Trans. Electromag. Compat., 26: 79-83; 1984.

[6] Shelkunoff, S.A.; Odarenko, T.M. Crosstalk between coaxial transmission lines. Bell Sys. Tech. J., 16: 144-164; 1937.

[7] Kuznetsov, P.I.; Stratonovich, R.L. The Propagation of Electromagnetic Waves in Multiconductor Transmission Lines. New York: Pergamon; 1964.

[8] Frankel, S. Multiconductor Transmission Line Analysis. Dedham, MA: Artech House; 1977.

[9] Gupta, K.C.; Garg, R.; Bahl, I.J. Microstrip Lines and Slotlines. Dedham, MA: Artech House; 1979. 
[10] Paul, C.R. Frequency response of multiconductor transmission lines illuminated by an electromagnetic field. IEEE Trans. Electromag. Compat., 18: 183-190; 1976.

[11] Paul, C.R.; Feather, A.E. Computation of the transmission line inductance and capacitance matrices from the generalized capacitance matrix. IEEE Trans. Electromag. Compat.; 18: 175-183; 1976.

[12] Wei, C.; Harrington, R.F.; Mautz, J.R.; Sarkar, T.K. Multiconductor transmission lines in multilayered dielectric media. IEEE Trans. Microwave Theory Tech., 32: 439-450; 1984.

[13] Hoffman, R.K. Handbook of Microwave Integrated Circuits. Norwood, MA: Artech House; 1987.

[14] Paul, C.R.; Everett, W.W. Printed circuit board crosstalk, Proc. Int. IEEE EMC Symp.: 452-459; 1985.

[15] Dunn, J.M.; Howard, L.C.; Larsen, K. An efficient algorithm for the calculation of parasitic coupling between lines in MICs. IEEE Trans. Microwave Theory Tech., to be published.

[16] Mosig, J. Integral equation technique. Chapter 4 in Numerical Techniques for Microwave and Millimeter-Wave Passive Structures, ed. by T. Itoh, New York: Wiley; 1989.

[17] Dunn, J.M. A uniform asymptotic expansion for the Green's functions used in microstrip caclulations. IEEE Trans. Microwave Theory Tech., 39: $1223-1226 ; 1991$.

[18] Harrington, R.F. Time-Harmonic Electromagnetic Fields. New York: McGraw-Hil1; 1961 .

[19] Weeks, W.L. Antenna Engineering. New York: McGraw-Hill; 1968.

[20] Cory, H.; Shiran, S. The importance of losses in the coupling of transmission 1ines. IEEE Trans. Electromag. Compat., 30: 567-570; 1988.

[21] Taylor, C.D.; Satterwhite, R.S.; Harrison, C.W. The response of a terminated two-wire transmission line excited by a nonuniform electromagnetic field. IEEE Trans. Antennas Propagat., 13: 987-989; 1965.

[22] Smith, A.A. Coupling of External Electromagnetic Fields To Transmission Lines. New York: Wiley; 1977.

[23] Yuan, Y.; Nyquist, D. Full-wave perturbation theory based upon electric field integral equations for coupled microstrip transmission lines. IEEE Trans. Microwave Theory Tech., 38: 1576-1584; 1990.

[24] Hammerstad, E.; Jensen, O. Accurate models for microstrip computeraided design. Proc. Int. IEEE MTT Symp.: 407-409; 1980. 
[25] Djordjevic, A.R.; Sarkar, T.K.; Harrington, R.F. Time-domain response of multiconductor transmission lines. Proc. IEEE, 75: 743-764; 1987.

[26] Rumsey, V.H. Frequency Independent Antennas. New York: Academic Press; 1966.

[27] Hill, D.A.; Adams, J.W.; Ma, M.T.; Ondrejka, A.R.; Riddle, B.F.; Crawford, M.L.; Johnk, R.T. Aperture excitation of electrically large, lossy cavities. Nat. Inst. Stand. Tech. (U.S.) Tech. Note 1361; 1993.

[28] Wheeler, H.A. Formulas for the skin effect. Proc. IRE, 30: 412-424; 1942.

[29] Pucel, R.A.; Masse, D.J.; Hartwig, C.P. Losses in microstrip. IEEE Trans. Microwave Theory Tech., 16: 342-350, 1968.

[30] Lee, H.Y.; Itoh, T. Phenomenological loss equivalence method for planar quasi-TEM transmission line with a thin normal conductor or superconductor. IEEE Trans. Microwave Theory Tech., 37: 1904-1909; 1989.

[31] Heinrich, W. Full-wave analysis of conductor losses on MMIC transmission lines. IEEE Trans. Microwave Theory Tech., 38: 1468-1472; 1990.

[32] Heinrich, W. The resistance of strip conductors embedded in a twodimensional field distribution. Proc. Int. IEEE MTT Symp.: 417-420; 1993.

APPENDIX A. EQUIVALENCE OF POTENTIAL AND TRANSMISSION LINE THEORIES

The purpose of this appendix is to show the equivalence of the potential source terms on the right sides of eqs (17a) and (17b) to the coupled transmission line source terms on the right sides of eqs ( $1 c$ ) and (1d). The conditions that we require are that the two lines be parallel and only weakly coupled (separation s should not be too small).

We first consider the scalar potential term $j \omega \mathrm{C}_{22} \mathrm{~V}\left(\zeta_{2}\right)$ on the right side of eq (17b). Using the expression for $V$ in eq (16a), we can write the potential term as

$$
j \omega C_{22} V\left(\zeta_{2}\right)=j \omega C_{22} \int_{1 \text { ine } 1} \rho_{1}\left(\zeta_{1}\right) g_{e}\left(\zeta_{1} ; \zeta_{2}\right) d \zeta_{1} \text {, }
$$


where we have suppressed the $y$ dependence of $g_{e}$. If we assume that $g_{e}$ decays rapidly as $15_{1}-5_{2} 1$ increases, then we can factor out the charge from the integral in eq (Al) and approximate the integral over 1 ine 1 by an integral from $-\infty$ to $\infty$ :

$$
j \omega C_{22} V\left(\zeta_{2}\right) \approx j \omega C_{22} \rho_{1}\left(\zeta_{2}\right) \int_{-\infty}^{\infty} g_{e}\left(\zeta_{1} ; \zeta_{2}\right) d \zeta_{1}
$$

The integral over $\zeta_{1}$ is the potential on line 2 due to a constant unit charge per unit length on line 1. Frankel [8] defines this quantity as the element $P_{21}$ of the potential matrix [P] which is the inverse of the usual capacitance matrix. This allows us to write eq (A2) as

$$
j \omega C_{22} V\left(\zeta_{2}\right) \approx j \omega C_{22} P_{21} \rho_{1}\left(\zeta_{2}\right)
$$

Using the definition of the capacitance matrix [8], we can write the charge $\rho_{1}$ on line 1 as

$$
\rho_{1}\left(\zeta_{1}\right)=C_{11} v_{1}\left(\zeta_{1}\right)+C_{12} v_{2}\left(\zeta_{1}\right) \approx c_{11} v_{1}\left(\zeta_{1}\right)
$$

where we have used the fact that the coupling is weak $\left(\left|C_{12}\right|\right.$ is small compared to $C_{11}$ ). Substituting eq (A4) into eq (A3), we have

$$
j \omega C_{22} V\left(\zeta_{2}\right) \approx j \omega C_{22} P_{21} C_{11} V_{1}\left(\zeta_{1}\right)
$$

From the inverse of the capacitance matrix, we can approximate $\mathrm{P}_{21}$ by $-C_{12} /\left(C_{11} C_{22}\right)$ if we again assume weak coupling $\left(C_{12}^{2} \ll C_{11} C_{22}\right)$. Then we can write eq (A5) in the desired final form:

$$
j \omega C_{22} V\left(\zeta_{2}\right) \approx-j \omega C_{12} V_{1}\left(\zeta_{2}\right)
$$


Equation (A6) confirms that the source terms on the right sides of eqs (1d) and (17b) are approximately equal and that the scalar potential source term is approximately to the mutual capacitance source term. The actual expression for the mutual capacitance is given in Appendix B for the example of circular wires above a ground plane.

We now consider the vector potential term, $-j \omega A\left(\zeta_{2}\right)$, on the right side of eq (17a). Using the expression for $\bar{A}$ in eq (16b), we can write the vector potential term as

$$
-j \omega A\left(\zeta_{2}\right)=-j \omega \int_{1 \text { ine } 1} I_{1}\left(\zeta_{1}\right) g_{m}\left(\zeta_{1} ; \zeta_{2}\right) d \zeta_{1} \text {, }
$$

where we have suppressed the $\mathrm{y}$ dependence of $\mathrm{g}_{\mathrm{m}}$. We have also used the fact that the two 1 ines are parallel $\left(\hat{\zeta}_{1} \cdot \hat{\zeta}_{2}=1\right)$. If we assume that $g_{m}$ also decays rapidly as $\left|5_{1}-5_{2}\right|$ increases, then we can factor out the current from the integral in eq (A7) and approximate the integral over line 1 by an integral from $-\infty$ to $\infty$ :

$$
-j \omega A\left(\zeta_{2}\right) \approx-j \omega I_{1}\left(\zeta_{2}\right) \int_{-\infty}^{\infty} g_{m}\left(\zeta_{1} ; \zeta_{2}\right) d \zeta_{1} .
$$

The integral over $\zeta_{1}$ is the flux linkage with line 2 due to a constant unit current on line 1 . This quantity is defined as the mutual inductance $L_{21}$ [8]. Using this definition, we can write eq (A8) in the desired final form:

$$
-j \omega A\left(5_{2}\right) \approx-j \omega L_{21} I_{1}\left(5_{2}\right)
$$

Equation (A9) confirms that the source terms on the right sides of eqs (1c) and (17a) are approximately equal and that the vector potential source term is approximately equal to the mutual inductance source term. The actual expression for the mutual inductance is given in Appendix B for the example of circular wires above a ground plane. 
APPENDIX B. TRANSMISSION LINE PARAMETERS FOR CIRCULAR WIRES

In general, the microstrip geometry of figure 1 does not permit a closed-form analysis, and numerical techniques are required to obtain the transmission line parameters [12]. The difficulties are that the strips support an unknown current or charge distribution that must be computed numerically and that the dielectric substrate leads to fairly complicated Green's functions, $g_{e}$ and $g_{m}[17]$. However, it is instructive to analyze a simple, circular-wire geometry to see how the various transmission line parameters and Green's functions are related.

Consider the two-wire transmission line geometry in figure 9. Identical circular wires of radius $a$ are located at a height $h$ and a separation $s$ above a perfectly conducting ground plane. The wires are also perfectly conducting. We consider the weak coupling case where we assume $s / h>1$. We also make the thin-wire assumption $(h / a>1)$.

We consider first the electrostatic problem which leads to the capacitance matrix [C]. Line 1 supports a uniform line charge per unit length $\rho_{1}$ and is at a potential $v_{1}$. Line 2 also supports a line charge per unit length $\rho_{2}$ and is at a potential $\mathrm{V}_{2}$. Following the formalism of Frankel [8], we write the voltage column vector [V] as the product of the potential matrix $[P]$ and the charge matrix $[\rho]$ :

$$
[V]=[P][\rho] .
$$

The potential matrix is the inverse of the capacitance matrix [C]:

$$
[P]=[C]^{-1} \text {. }
$$

The elements of the potential matrix are [8]

$$
P_{11}=P_{22} \approx \frac{1}{2 \pi \epsilon_{0}} \ln (2 h / a)
$$

and 


$$
\mathrm{P}_{12}=\mathrm{P}_{21} \approx \frac{1}{2 \pi \epsilon_{0}} \ln (\mathrm{d} / \mathrm{s})
$$

where $d=\left[s^{2}+(2 h)^{2}\right]^{1 / 2}$. Since we have assumed that $s / h \gg 1$, we can simplify (B3b) to

$$
\mathrm{P}_{12}=\mathrm{P}_{21} \approx \frac{\mathrm{h}^{2}}{\pi \epsilon_{0} \mathrm{~s}^{2}} .
$$

The elements of the capacitance matrix are obtained from the inverse of $[P]$ :

$$
C_{11}=C_{22}=P_{11} /\left(P_{11}^{2}-P_{12}^{2}\right) \approx 1 / P_{11}
$$

and

$$
C_{12}=C_{21}=-P_{12} /\left(P_{11}^{2}-P_{12}^{2}\right) \approx-P_{12} / P_{11}^{2} \text {. }
$$

Substituting eqs (B3) and (B4) into (B5), we obtain

$$
C_{11}=C_{22} \approx \frac{2 \pi \epsilon 0}{\ln (2 h / a)}
$$

and

$$
C_{12}=C_{21} \approx \frac{-4 \pi \epsilon h^{2}}{s^{2} \ln ^{2}(2 h / a)} .
$$

It is also interesting to show how the coupling terms $\left(P_{12}-P_{21}\right)$ in the potential matrix can be derived from the integral of the Green's function $g_{e}$. We can write the Green's function in the following form:

$$
g_{e}\left(5_{1}, 5_{2}\right)=\frac{1}{4 \pi \epsilon_{0}}\left(r^{-1}-r_{i}^{-1}\right)
$$

where

$$
r=\left[s^{2}+\left(5_{2}-5_{1}\right)^{2}\right]^{1 / 2} \text { and } r_{i}-\left[s^{2}+(2 h)^{2}+\left(5_{2}-5_{1}\right)^{2}\right]^{1 / 2} \text {. }
$$


Then we can write $\mathrm{P}_{21}$ as the following integral:

$$
\mathrm{P}_{21} \approx \int_{-\infty}^{\infty} \frac{1}{4 \pi \epsilon_{0}}\left(\mathrm{r}^{-1}-\mathrm{r}_{\mathrm{i}}^{-1}\right) \mathrm{d} \zeta_{1}=\frac{1}{2 \pi \epsilon_{0}} \ln (\mathrm{d} / \mathrm{s})
$$

which is in agreement with Frankel's result [8] in eq (B3b).

It is also useful to perform the integral in eq (B8) over finite limits, - I to $\mathrm{L}$, to determine what length of the transmission line actually contributes significantly to the coupling represented by $\mathrm{P}_{21}$. Thus we define $\mathrm{P}_{21 \mathrm{~L}}$ as the following finite integral:

$$
\mathrm{P}_{21 \mathrm{~L}}=\int_{-\mathrm{L}}^{\mathrm{L}} \frac{1}{4 \pi \epsilon_{0}}\left(\mathrm{r}_{0}^{-1}-\mathrm{r}_{01}^{-1}\right) \mathrm{d} \zeta_{1},
$$

where

$$
r_{0}=\left(s^{2}+s_{1}^{2}\right)^{1 / 2} \text { and } r_{0 i}=\left[s^{2}+(2 h)^{2}+5_{1}^{2}\right]^{1 / 2}
$$

This integral can be evaluated in terms of logarithmic functions and approximated to the following form:

$$
\mathrm{P}_{21 \mathrm{~L}} \approx \frac{\mathrm{h}^{2}}{\pi \epsilon_{0} \mathrm{~d}^{2}}\left(1-\frac{\mathrm{s}^{2}}{2 \mathrm{~L}^{2}}\right) \approx \mathrm{P}_{21}\left(1-\frac{\mathrm{s}^{2}}{2 \mathrm{~L}^{2}}\right)
$$

For $\mathrm{P}_{21 \mathrm{~L}}$ to be approximately equal to $\mathrm{P}_{21}$, we need the second term in the parentheses to be small compared to 1 . This yields

$$
\mathrm{L}^{2} / \mathrm{s}^{2} \gg 1 / 2
$$

The physical interpretation of eq (B11) is that the capacitive coupling to a given point on a parallel line occurs primarily from the charge over a length $2 \mathrm{~L}$ approximately equal to $2 \mathrm{~s}$. 
Because the inductance matrix [L] is closely related to the potential and capacitance matrices, we can obtain the elements from the previous analyses. The simplest relationship involves the potential matrix [8]:

$$
[L]=\mu_{0} \epsilon_{0}[P]
$$

From eqs (B3a) and ( $B 12$ ), the diagonal elements, $\mathrm{L}_{11}$ and $\mathrm{L}_{12}$, are

$$
\mathrm{L}_{11}=\mathrm{L}_{22}=\mu_{0} \epsilon_{0} \mathrm{P}_{11} \approx \frac{\mu_{0}}{2 \pi} \ln (2 \mathrm{~h} / \mathrm{a})
$$

From eqs (B4) and (B12), the coupling elements, $\mathrm{L}_{12}$ and $\mathrm{L}_{21}$, are

$$
L_{12}=L_{21}=\mu_{0} \epsilon_{0} P_{12} \approx \frac{\mu_{0} h^{2}}{\pi s^{2}}
$$

The derivation of $\mathrm{L}_{12}$ by integrating the magnetic Green's function $\mathrm{g}_{\mathrm{m}}$ over a line with either infinite or finite limits follows the same mathematics as the electrostatic case in eqs (B7)-(B11) and will not be repeated.

APPENDIX C. SCALE MODELS

For crosstalk applications involving small microstrip lines, measurements are more conveniently done on larger scale models, such as the coupled microstrip lines pictured in figure 4. For the time-harmonic form of Maxwell's equations, scaling of frequency and length in nondispersive, lossless media is well known. For the example of frequency-independent antennas [26], "the entire electrical performance is frequency-independent if all length dimensions are scaled in proportion to frequency."

Now consider a lossy, inhomogeneous medium (such as a small microstrip line), characterized by position-dependent permittivity $\epsilon(\bar{r})$, permeability 
$\mu(\bar{r})$, and conductivity $\sigma(\bar{r})$. If we wish to scale up in size by a factor $S$, then the new (primed) parameters need to be related to the original (unprimed) parameters by [27]

$$
\begin{aligned}
& \bar{r}^{\prime}=S \bar{r}, \\
& \omega^{\prime}=\omega / S, \\
& \sigma^{\prime}\left(\bar{r}^{\prime}\right)=\sigma(\bar{r}) / S, \\
& \epsilon^{\prime}\left(\bar{r}^{\prime}\right)=\epsilon(\bar{r}),
\end{aligned}
$$

and

$$
\mu^{\prime}\left(\bar{r}^{\prime}\right)=\mu(\bar{r})
$$

The length and frequency scaling is as expected, and keeping the permeability $\mu^{\prime}$ equal to the original $\mu$ is automatic for nonmagnetic materials. Keeping the substrate permittivity constant can be done approximately for low-dispersion dielectrics, but the main difficulty comes in scaling the microstrip and ground-plane conductivities. These conductivities of the scale model should be multiplied by a factor $1 / \mathrm{S}$. (This means a decrease for $\mathrm{S}>1$.)

The required decrease in conductivity for the metal strips and ground plane obviously represents a materials problem unless a different metal with the required lower conductivity can be used. Normally this is not practical because it more convenient to use copper in both the original and scale model. Another possibility would be to adjust the thickness of the strips and ground plane to obtain the desired resistance $R$ per unit length. The scaled resistance $R^{\prime}$ per unit length should equal the original resistance $R$ per unit length times $1 / S$. The difficulty in controlling $R^{\prime}$ by adjusting metal thickness is that $R^{\prime}$ depends on thickness in a rather complicated manner and is strongly dependent on the ratio of skin depth to thickness. If the metal conductivity and thickness are both scaled according to eq 
(C1), the ratio is maintained because both the thickness and skin depth increase by a factor $S$ upon scaling.

If the metal conductivity is not scaled, then a good loss theory is required to make the proper adjustment in metal thickness. The present choices are between approximate theories, such as the incremental inductance rule $[28,29]$ or the phenomenological loss equivalent method [30], and fullwave numerical methods $[31,32]$. More work is needed in this area. 


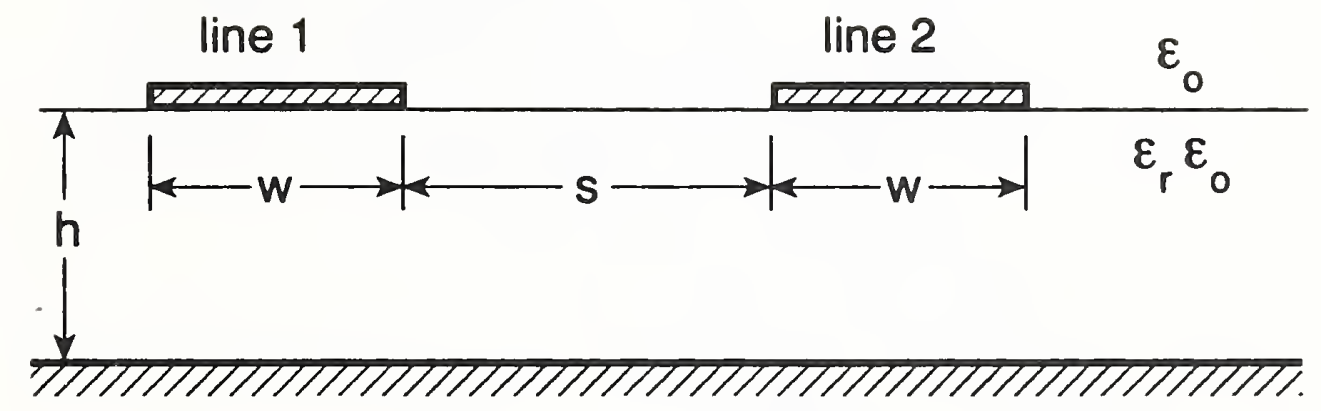

Figure 1. Cross-sectional geometry for a pair of identical microstrip transmission lines. 


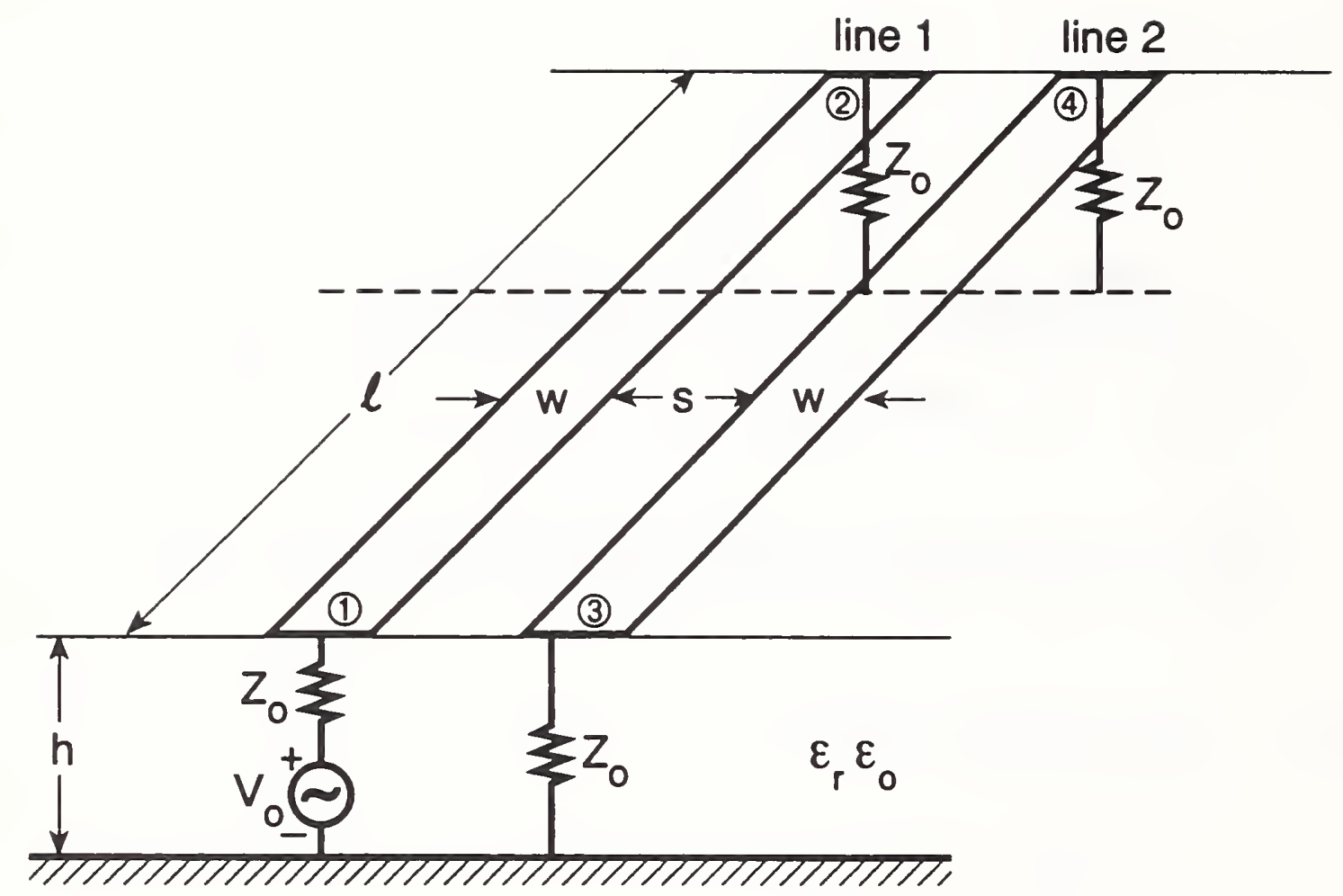

Figure 2. Two identical microstrip lines terminated in the characteristic impedance $Z_{0}$ of an isolated line. Line 1 is excited at port 1 . 

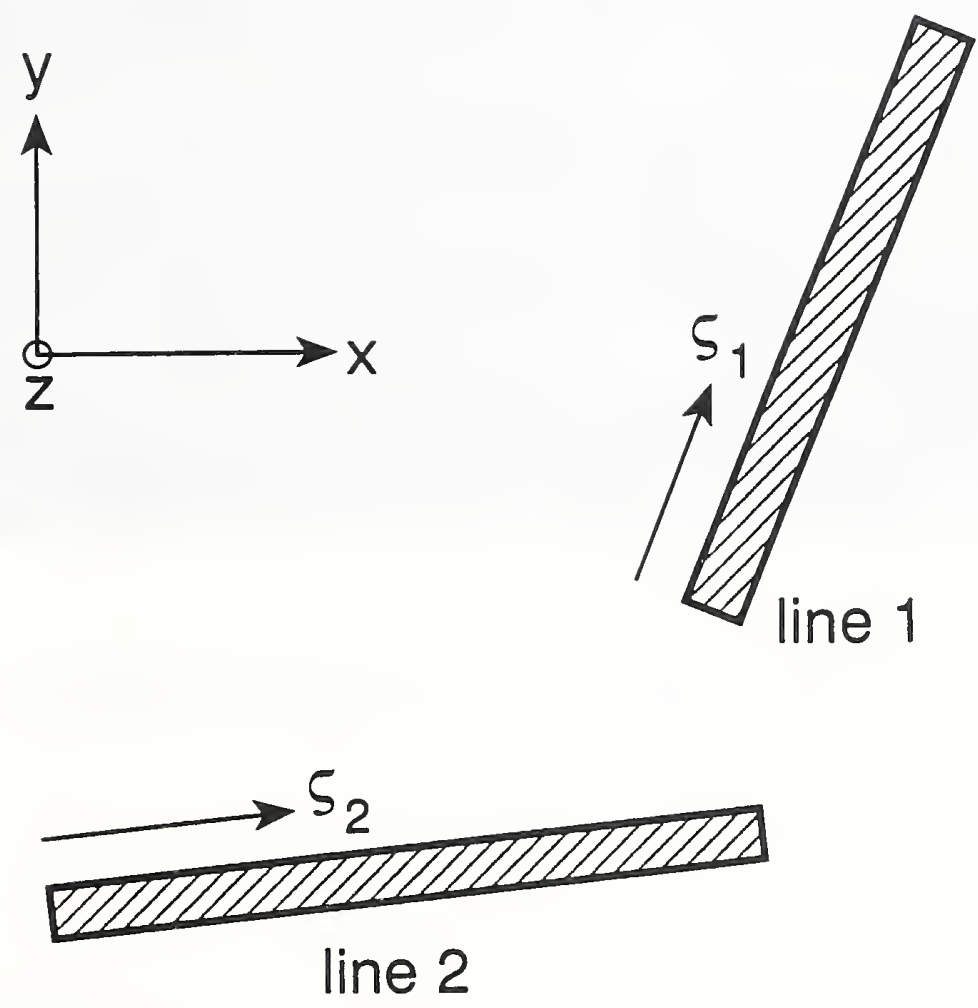

Figure 3. Two microstrip lines of arbitrary orientation located on the same substrate. 


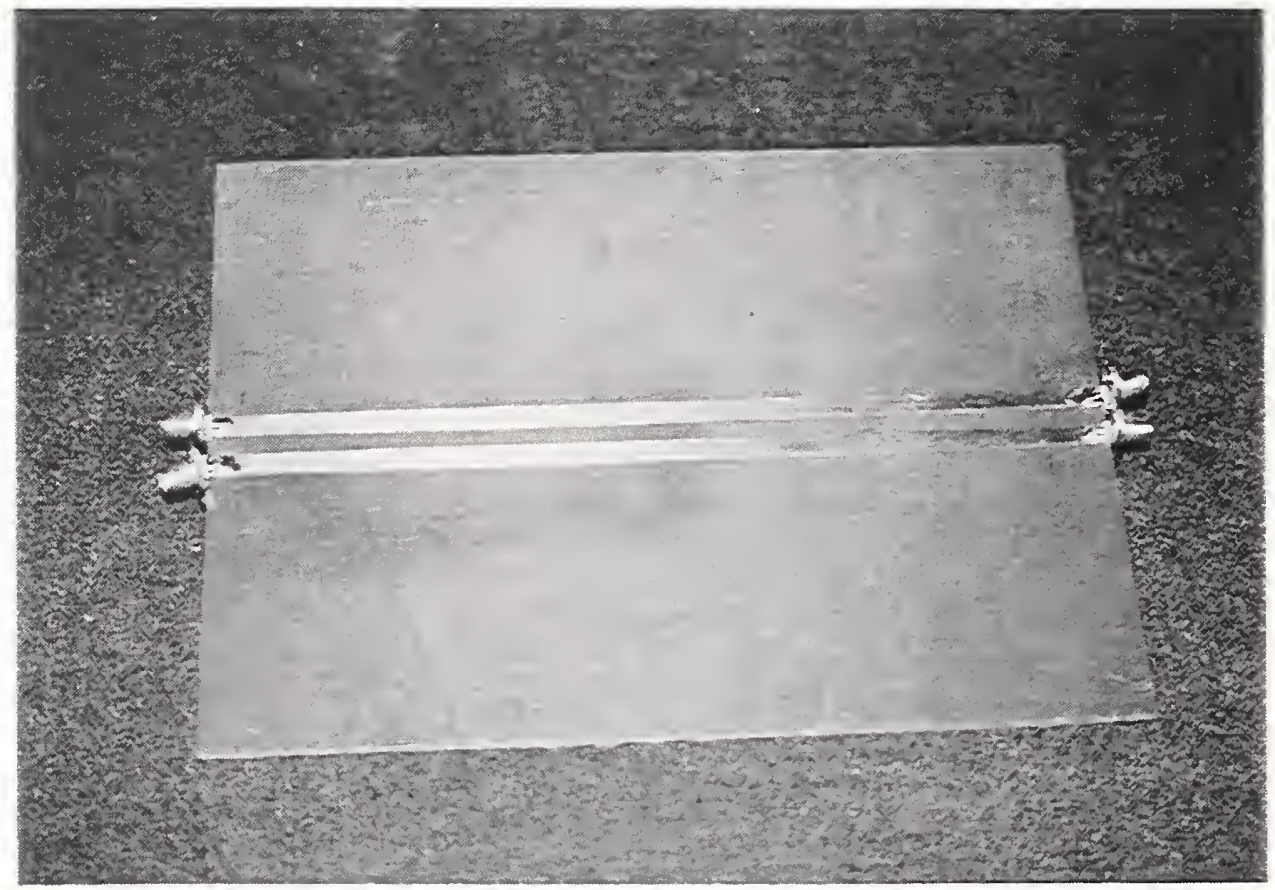

Figure 4. Two identical microstrip lines with coaxial connectors. 


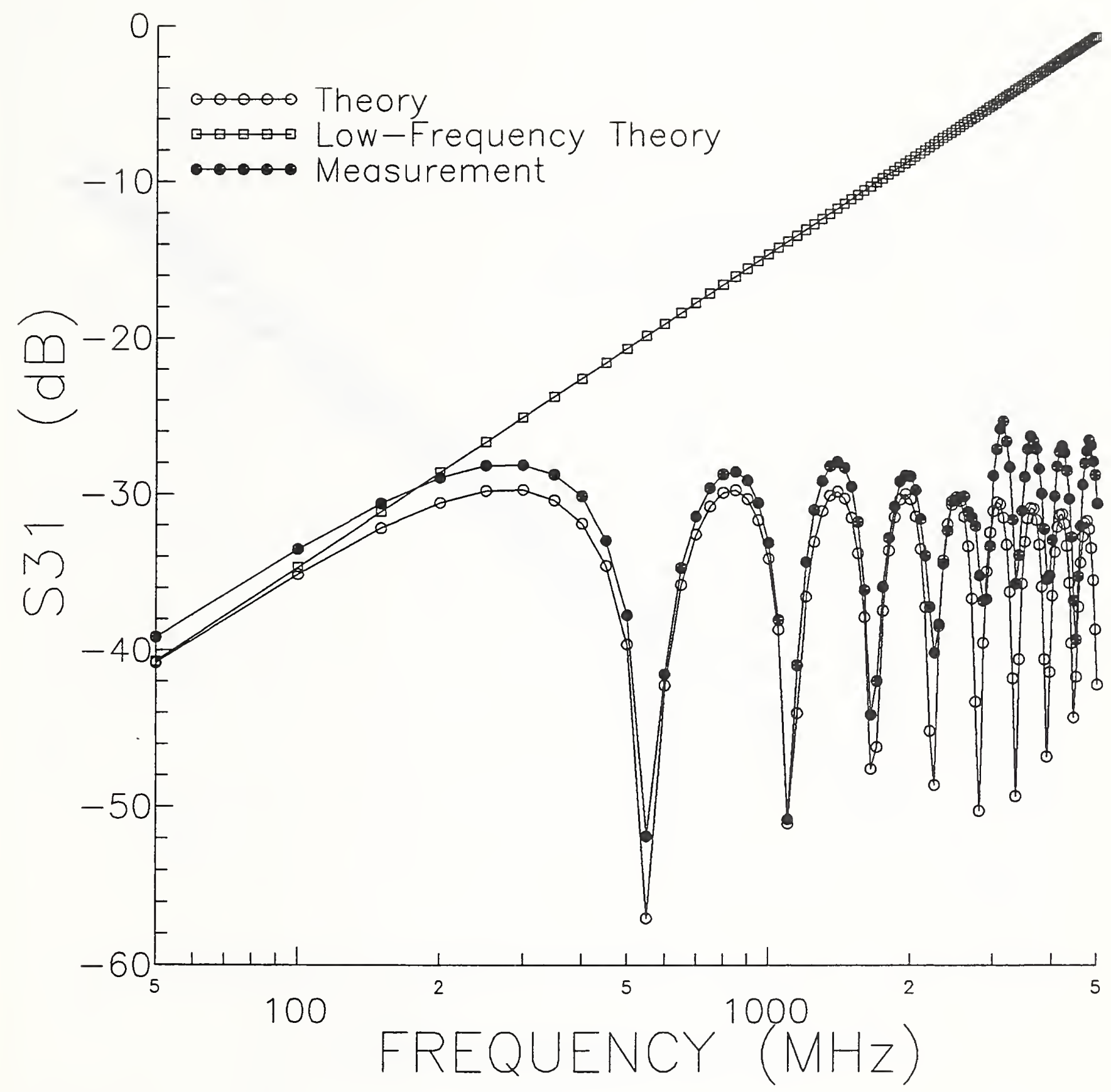

Figure 5. Magnitude of $\mathrm{S}_{31}$ (near-end crosstalk) from theory and measurement. 


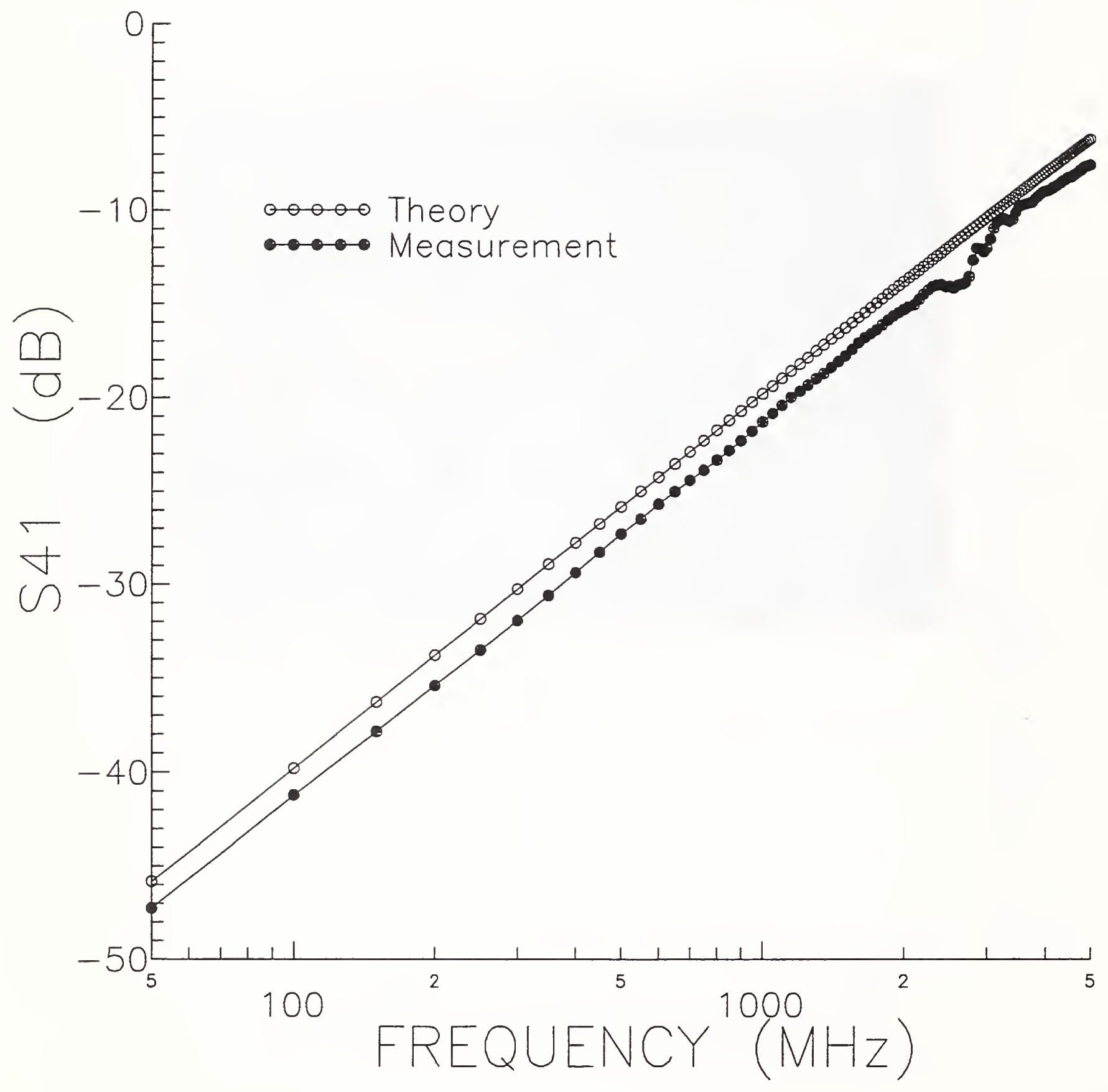

Figure 6. Magnitude of $\mathrm{S}_{41}$ (far-end crosstalk) from theory and measurement. Results for the low-frequency theory are not shown because they are essentially identical to the more general theory. 


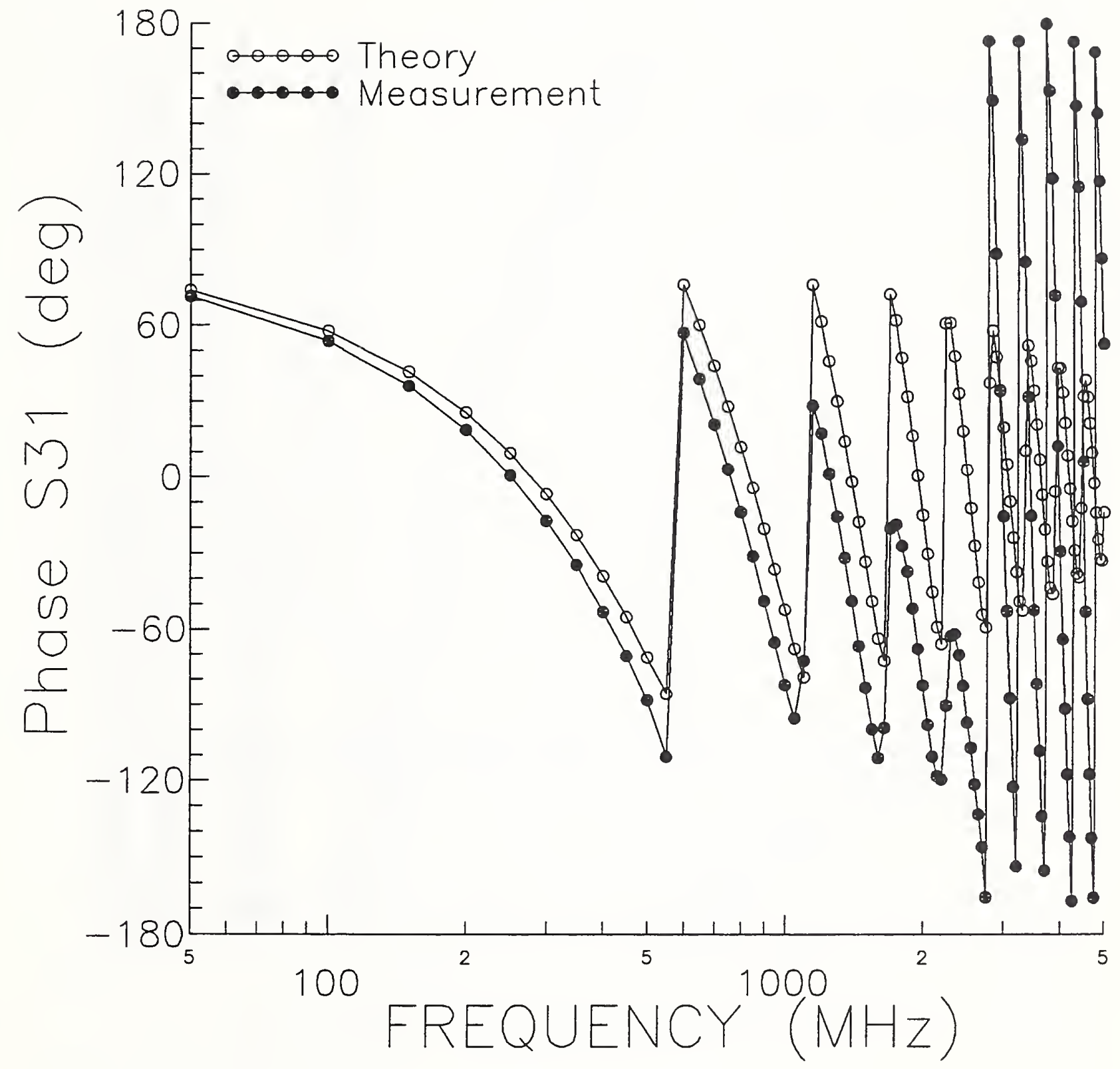

Figure 7. Phase of $\mathrm{S}_{31}$ (near-end crosstalk) from theory and measurement. 


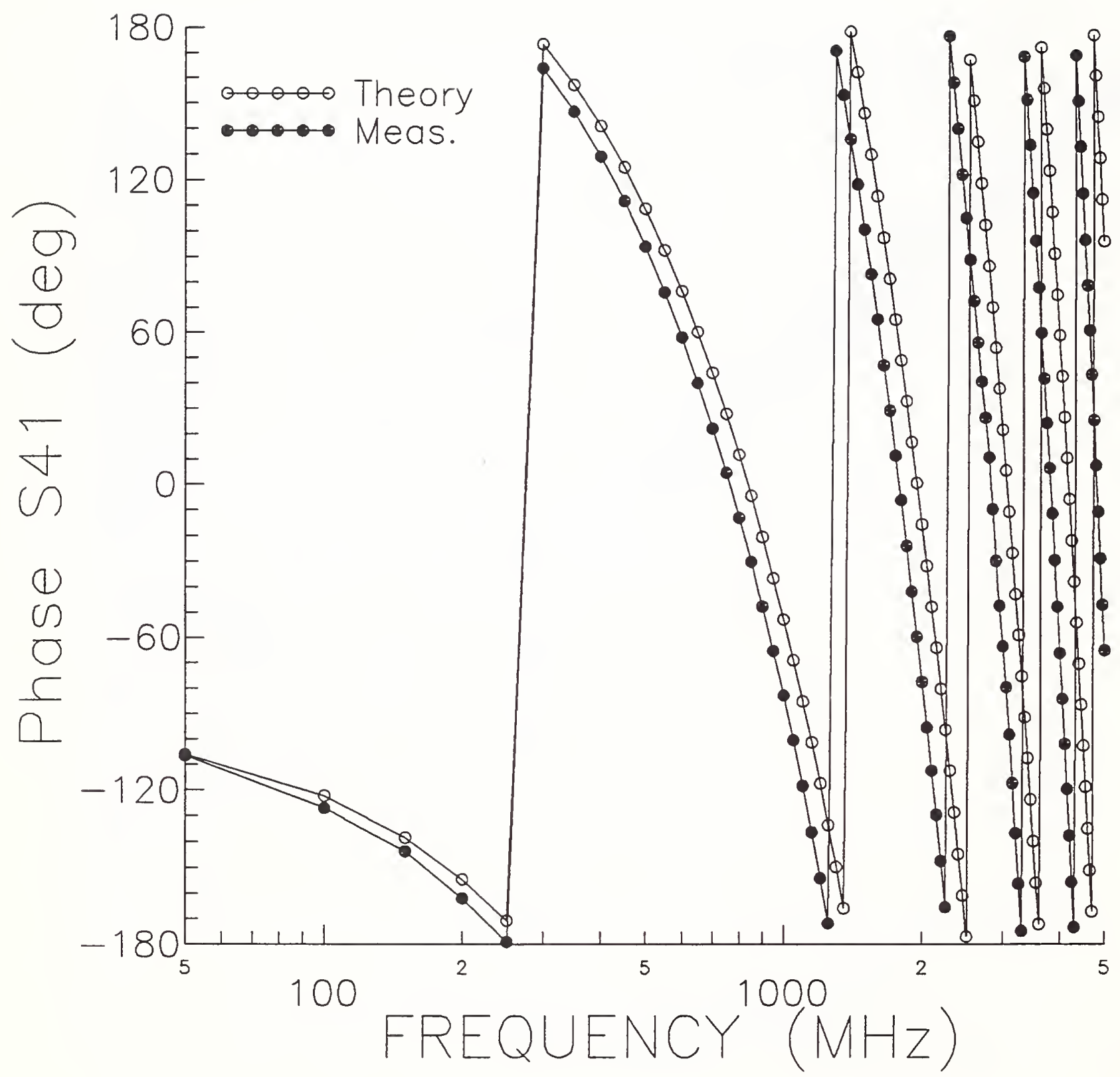

Figure 8. Phase of $\mathrm{S}_{41}$ (far-end crosstalk) from theory and measurement. 


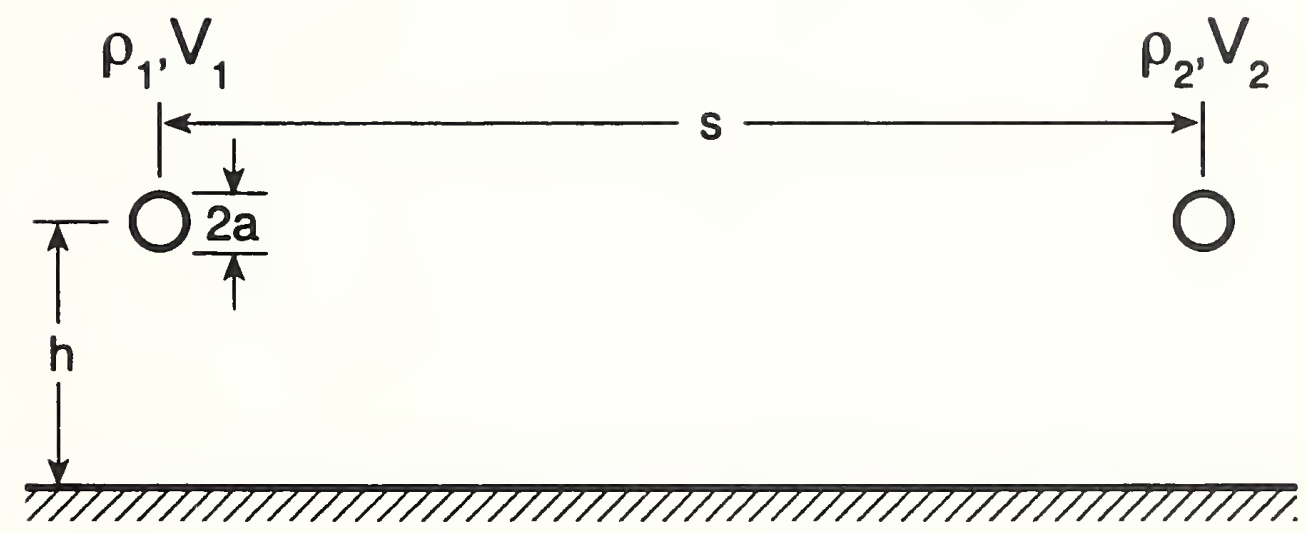

Figure 9. Two identical circular wires located in free space over a perfect ground plane. 


